\title{
Frontières
}

\section{Le politique et la mort}

\section{Joseph J. Lévy et Pascal Hintermeyer}

Volume 19, numéro 1, automne 2006

Enjeux politiques et mort

URI : https://id.erudit.org/iderudit/016628ar

DOI : https://doi.org/10.7202/016628ar

Aller au sommaire du numéro

Éditeur(s)

Université du Québec à Montréal

ISSN

1180-3479 (imprimé)

1916-0976 (numérique)

Découvrir la revue

Citer ce document

Lévy, J. J. \& Hintermeyer, P. (2006). Le politique et la mort. Frontières, 19(1), 6-8. https://doi.org/10.7202/016628ar

Ce document est protégé par la loi sur le droit d'auteur. L'utilisation des services d'Érudit (y compris la reproduction) est assujettie à sa politique d'utilisation que vous pouvez consulter en ligne.

https://apropos.erudit.org/fr/usagers/politique-dutilisation/
Cet article est diffusé et préservé par Érudit.

Érudit est un consortium interuniversitaire sans but lucratif composé de l’Université de Montréal, l'Université Laval et l'Université du Québec à Montréal. Il a pour mission la promotion et la valorisation de la recherche. https://www.erudit.org/fr/ 


\section{Le politique et la mort}

\section{Joseph J. Lévy, \\ professeur, Université du Québec à Montréal. \\ Pascal Hintermeyer \\ professeur, Université Marc-Bloch, Strasbourg.}

Le politique, qui renvoie aux modalités de la gouvernance d'un État ou d'une collectivité, n'est pas sans être préoccupé par les enjeux touchant la question de la mort et de sa gestion sociale. C'est à cette problématique complexe que le présent numéro de Frontières est consacré. Il regroupe ainsi des contributions de chercheurs et auteurs en sciences humaines qui éclairent l'un ou l'autre de ces enjeux.

Dans les responsabilités gouvernementales des sociétés, la gestion de l'ordre public et la sécurité des citoyens sont associées au principe du monopole de la violence légitime. La force publique est la seule, théoriquement, à pouvoir porter et utiliser les armes, dans des conditions spécifiques, même si des entorses à cette règle sont fréquentes. Les autorités étatiques sont aussi responsables d'enquêter sur les morts violentes et de traduire en justice les coupables, le châtiment pouvant aller jusqu'à la peine capitale dans certains pays. Si, dans plusieurs États, la peine de mort a été abolie, elle continue d'être pratiquée dans des pays comme les États-Unis, par exemple, et ce, sous des formes variées, de la chaise électrique à la chambre à gaz. Les mouvements visant à l'abolition de ces pratiques tendent à se multiplier et des organismes non gouvernementaux à l'échelle mondiale, comme Amnistie internationale, mènent des campagnes pour éliminer cette forme dépassée de sanction légale.

Deux articles dans ce numéro mettent en relief les politiques et les débats entourant cette question. Emmanuel Taïeb examine ainsi le processus de dépublicisation des exécutions capitales qui survient en France en 1939 pour se poursuivre dans un espace plus fermé, celui des cours des prisons. Ce processus s'inscrit, d'une part, dans une politique fondée sur l'«arraisonnement pénitentiaire » et, d'autre part, dans une transformation des sensibilités qui vise à occulter les scènes de violence et de mort, sans cependant éliminer cette sanction.

Prolongeant cette analyse, Raphaël Micheli s'attache à l'analyse d'un corpus de discours parlementaires français sur l'abolition de la peine de mort, en mettant en évidence «comment - au plan argumentatif - les orateurs parviennent à passer de la reconnaissance d'une pulsion mortifère à l'affirmation de l'«impossibilité d'une justice de mort ». L'institution judiciaire n'a pas une fonction thérapeutique et ne peut pas reprendre à son compte le lexique de la vengeance ou le référent de la mort, qui ne peuvent constituer une propriété de la justice. Cette analyse met en relief les ruptures entre les plans individuel ou interindividuel et supra-individuel, mais aussi la sollicitude à l'égard des familles des victimes - une catégorie sociale absente des discours abolitionnistes antérieurs et qui apparaît comme l'expression d'une nouvelle sensibilité sociale et politique.

L'intervention de l'État se fait aussi sentir au plan de la protection de la santé publique, à travers l'établissement des politiques de salubrité et d'hygiène, de prévention, du traitement et du contrôle des épidémies. La définition des critères expérimentaux et éthiques précis auxquels les essais pharmacologiques doivent obéir et qui ont pour objectif la réduction des risques pour les individus constitue un autre champ d'application de la prévenance politique. Les lois concernant l'avortement et le recours à l'euthanasie suggèrent que l'État intervient sur la régulation de la natalité et de la mortalité. Par ailleurs, les modalités d'inhumation ou de traitement des cadavres obéissent à des règlements administratifs spécifiques et variables selon les sociétés, alors que la définition même de la mort répond à des critères biomédicaux complexes, associés au développement technologique, et qui interviennent dans les procédures de transplantation des organes.

Pascal Hintermeyer, dans sa réflexion sur les rapports entre politique et mort à partir d'une perspective anthropologique, souligne la mise en place des valeurs de protection de la vie et de la gestion de la violence, de même que les répercussions des révolutions techniques et scientifiques qui, à partir du XVII ${ }^{\mathrm{e}}$ siècle, ont contribué à un « reflux de la mort». Les usages de la mort au profit de la vie, les manipulations des cadavres et la transplantation d'organes dans le champ biomédical témoignent de ces tendances.

Serenella Nonnis Vigilante illustre la prégnance des préoccupations de santé publique dans le traitement des cadavres en analysant les pratiques mortuaires sur les champs de bataille de la Première Guerre mondiale. Afin d'éviter les épidémies, il fallait disposer des corps le plus rapidement possible, soit par enterrement sommaire, inhumation dans des fosses ou crémation combinant des produits chimiques et le feu. Ces stratégies ont contribué à effacer l'identité des cadavres et à empêcher le repérage des tombes, autant d'élément qui concourent à un travail de deuil difficile sinon impossible pour les familles éplorées. 
L'absence de sollicitude étatique à l'égard de ses citoyens peut aller à d'autres extrêmes, comme ce fut le cas dans l'Allemagne nazie, avec ses politiques d'euthanasie pour des motifs eugéniques à l'égard des personnes souffrant de handicaps ou de maladies mentales. La répression étatique à des fins de contrôle politique peut aller jusqu'à l'élimination des groupes d'opposants par des moyens violents (assassinats politiques, exécutions extrajudiciaires, disparitions, camps de la mort, goulags, génocides) ou employer des stratégies plus subtiles. Christian Saint-Germain nous invite à une réflexion critique sur les arrimages existant entre les luttes contre les pandémies et les groupes subversifs dans le monde contemporain. Elles constitueraient les deux faces d'une perspective totalitaire contrôlée par l'État dont la stratégie essentielle est «de savoir inquiéter» et d'amener ainsi les citoyens à accepter un dispositif qui repose sur la menace constante, tout en prétendant se fonder sur la sécurité. Ce système, qui alimente la peur, contribue à les prendre en otages et à imposer un train de lois qui limitent les libertés et renforcent la mise en place d'un État policier et panoptique dans une société où le lien social se délite. Ainsi «virus et terroristes marquent l'infiltration de l'insensé dans le dispositif de planification rationnelle des sociétés de consommation » dont l'horizon apparaît singulièrement problématique.

Les guerres civiles et interétatiques constituent un autre exemple de l'arrimage complexe entre politique et mort. Les guerres mondiales, ainsi que les nombreux foyers de guerre aujourd'hui présents dans le monde, mettent en évidence le fait que la guerre est la continuation de la politique par d'autres moyens, comme l'écrivait Von Clausewitz. Les conventions internationales qui régissent les conflits sont censées protéger les populations civiles, mais ces dernières se retrouvent souvent prises en otage, massacrées ou confrontées à des nettoyages ethniques et à des génocides. Plusieurs articles dans ce numéro traitent de cette problématique à partir d'angles divers. Ainsi, à partir des textes journalistiques du célèbre écrivain George Orwell sur la guerre civile espagnole, Louis Gill décrit les stratégies utilisées par l'Union soviétique, pour éliminer les forces de gauche, trotskystes et anarcho-syndicalistes jugées comme une menace à la Révolution. Les ressemblances entre les méthodes employées par les fascistes espagnols et les systèmes politiques nazi et soviétique soulignent la parenté idéologique entre ces mouvements qui n'hésitaient pas à recourir à l'assassinat des figures d'opposition, une stratégie qui sera abandonnée au profit de méthodes plus extrêmes liées aux exterminations de masse.

Dans son analyse des camps soviétiques et nazis, Luba Jurgenson pointe les différences dans les finalités entre ces deux systèmes fondés, le premier sur la rééducation et sur la production des cadavres rendus à l'État qui effaçait leur identité, le second sur un projet plus total, celui de la destruction du résidu même que constituait le cadavre. Dans ces conditions, les rituels, minimes, entourant la mort des détenus obéissent à des référents différents. Jurgenson montre comment dans les camps nazis, l'interdit de dire la mort vise à disculper le meurtrier: "Pas de destruction, donc pas de criminel ni de victime. » En l'absence de sépultures, c'est le texte du témoin qui le remplace, comme l'illustrent les exemples littéraires d'auteurs russes, le «texte-sépulture » qui permet ainsi «la transmission de l'expérience des morts aux vivants».

La littérature n'est pas le seul véhicule qui permet cette transmission. Dans des documentaires tels que Nuit et Brouillard ou des films de fiction comme L'aveu ou Amen de Costa-Gavras, le cinéma a contribué à rendre compte des cruautés de notre siècle. À partir du documentaire S21, la machine de mort khmère rouge, de Rithy Panh, Mouloud Boukala présente le cinéma comme «une machine [qui] tente de comprendre les mécanismes d'une autre machine, une machine à fabriquer des bêtes », en faisant témoigner des tortionnaires du bureau 21 et des rescapés sur les techniques de torture utilisées pour systématiquement briser les prisonniers et obtenir des preuves inventées qui conduisaient à leur exécution. La technique filmique, qui fait du spectateur un investigateur attentif aux détails que la caméra collecte, fait aussi appel à la réactivation des gestes des tortionnaires, rendant visibles leur violence et leur cruauté. Ce documentaire, présenté au Cambodge, a permis de briser le tabou du silence et de resituer le génocide dans l'espace collectif, un geste éminemment politique dans sa portée.

Dans un autre contexte, celui de l'Argentine, Alice Verstraeten analyse les témoignages de familles qui ont connu la disparition d'un de leurs proches à cause de l'emprise féroce de la junte militaire entre 1976 et 1983. Elle met en évidence les stratégies sous-jacentes à la terreur et les techniques de liquidation brutales des opposants. Compte tenu de ces conditions, les familles ne peuvent vivre leur deuil de façon normale et se retrouvent maintenues dans un état de liminalité prolongé. La notion de disparition en vient à signifier de façon prédominante cette condition, mais elle reste insuffisante et inadéquate pour rendre compte d'un processus où les individus ont été «tués, retués et contretués » par le déni du crime. Les différentes modalités de revendication et d'identification des corps peuvent alors réparer le tissu familial et social et reconstruire une nouvelle éthique politique.

L'État participe par ailleurs au travail de deuil et de mémoire rattaché aux conflits en définissant les dates de commémoration et les rituels qui les accompagnent, en contribuant à l'érection et à l'entretien des monuments aux morts et des nécropoles, en soutenant les musées et les centres de recherche qui contribuent à la construction du passé et à son interprétation. On voit ainsi se développer un tourisme de mémoire dans les hauts lieux des conflits comme les champs de bataille, les plages de débarquement, les cimetières de guerre, les musées, les lieux de mémoire dans les villes. Faisant un retour historique sur l'occupation nazie de la ville de Lyon, Armelle Chitrit nous invite à suivre un itinéraire commenté de cette capitale de la Résistance française et de ses figures centrales, notamment celle de Jean Moulin. Associant mémoire historique et textes poétiques d'écrivains de cette époque, elle rappelle notamment la situation des Juifs et la déportation de plusieurs d'entre eux.

La disparition des grands de ce monde, tragique ou attendue, fait aussi l'objet de mises en scène spectaculaires où l'expression $\mathrm{du}$ deuil public se conjugue aux intrigues politiciennes pour signifier les questions du pouvoir et de la légitimité. Pierre-Yves Beaudot analyse les mécanismes entourant l'organisation des funérailles des présidents de la République française entre 1877 et 1996, montrant comment elles sont le produit de négociations entre différentes instances, la famille, les héritiers politiques, le gouvernement, les officiants, en charge de l'organisation des funérailles et les célébrants, sans donner nécessairement lieu à des funérailles nationales. À partir d'exemples historiques, il présente les variations dans les modalités funéraires, mettant en évidence les influences religieuses catholiques. Les cérémonies apparaissent ainsi comme des «coproductions» dépendantes de plusieurs facteurs (époque, forces politiques, biographie du défunt, etc.) qui sont chaque fois réinterprétés en fonction de conditions particulières. Cette analyse révèle comment les dernières volontés du défunt sont reprises et transformées.

Emmanuel Fureix s'est, quant à lui, penché sur les funérailles publiques (funérailles d'État ou de l'opposition) qui prennent place entre 1814 et 1840 en France pour montrer leurs fonctions dans la socialisation politique. Il dégage le rôle du sentiment et de la sensibilité dans la construction du lien social et politique. Le deuil et les larmes collectifs deviennent l'expression du lien politique (royauté ou nation souveraine) alors que l'analyse des stratégies politiques autour des funérailles nationales indique bel et bien 
l'existence d' «un modèle parisien de la mort en politique à l'âge romantique», qui constitue «l'apprentissage de la représentation par le culte des morts, érigé en langage politique».

Cette pédagogie politique, Pascal Hintermeyer la retrouve aussi dans les stratégies liées aux mécanismes de consécration à des fins de réaffirmation des valeurs collectives (apposition des noms des disparus célèbres, portraits, statues dans les divers lieux de mémoire). Les exemples de personnages politiques au charisme reconnu ou dont les vicissitudes de l'existence, l'affrontement de la maladie ou la capacité de résister à l'adversité politique démontrent leur force morale et leur ténacité face à la mort, permettent aussi de faire consensus autour de leur personne.

La biographie de Zumbi, esquissée par Francine Saillant et Ana Lucia Araujo, illustre bien ces mécanismes de réappropriation d'une figure mythique et historique et sa fonction politique dans le contexte brésilien où, malgré la fin de l'esclavage, la contribution afro-brésilienne continue d'être occultée. C'est dans les années 1970 que le Zumbi commence à devenir un symbole de résistance du mouvement noir brésilien associé à des revendications pour l'égalité. Le 20 novembre, date de sa mort, est ainsi devenu journée nationale de la conscience noire, rappelant la «mémoire de résistance qui accompagne les vivants».

À l'inverse de cette figure dont le nom est revendiqué, Claude Javeau, dans son étude portant sur la figure du Soldat inconnu met en évidence une forme de mémorisation où l'absence de nom est le garant de l'identification nationale mise en scène par les rituels publics officiels. Cet attachement au Soldat inconnu met par ailleurs en relief l'expression de la souffrance féminine, celle des Mères, qui reprend des figures antérieures, comme celle de la mater dolorosa, ou la Marianne de la République et qui sont la métaphore de la Patrie. Le Soldat inconnu représenterait quant à lui la métonymie de l'ensemble des soldats morts, à l'héroïsme obscur, ordinaire. Reprenant la notion de «piaculaire» chez Durkheim qui l'associe à des fêtes tristes, Javeau analyse les rituels associés au Soldat inconnu en montrant comment il rejoint par là la société des disparus et contribue à l'expression des sentiments de repentir. Le tombeau continue donc de jouer un rôle significatif comme lieu de mémoire, même si l'on constate l'effacement de ces référents dans la conscience des citoyens.

Cette fascination pour le culte des morts n'est pas étrangère à certaines formes de tourisme contemporain, comme le signale Franck Michel, chez les Toraja d'Indonésie. Il dégage, dans un premier temps, les rapports complexes entre leur religion locale et les autres religions, d'une part, et les processus historiques, d'autre part, qui ont mené à l'intégration de la région toraja à l'espace politique indonésien et à sa modernisation. Il analyse ensuite les stratégies politiques associées au tourisme religieux attiré par les rituels et cérémonies funéraires élaborées qui se retrouvent dans cette collectivité. Ces stratégies ont donné lieu à une folklorisation des rites qui a entraîné un enrichissement et une reformulation de leurs traditions mortuaires, mais aussi un effritement des fondements de leur culture traditionnelle.

La célébration des morts, que rapporte Nicoletta Diasio, dans son étude sur les mémoires et les morts dans la Pologne postcommuniste, constitue aussi un moment fort du calendrier liturgique polonais, incluant les figures intellectuelles, artistiques et politiques révérées. Dans ce contexte, le travail de mémoire n'échappe pas non plus aux influences du politique. En recomposition constante, il met en relief les multiples récits qui se nourrissent d'évènements historiques choisis dans le répertoire antérieur à la Pologne contemporaine. L'émergence de multiples mémoires jusque-là réprimées donne lieu à une «effervescence mémorielle » où « [...] la mémoire collective ne se présente pas comme un artifice politique, établi a posteriori, mais comme un fleuve vivant qui modifie, anticipe, prépare les transformations ».

La question de la mémoire, mais surtout du témoignage est aussi présente dans l'œuvre de Primo Lévi, mort il y a vingt ans et dont Joseph Josy Lévy célèbre la mémoire. À cet effet, l'auteur relève, dans l'œuvre de ce témoin de la Shoah, des réflexions pertinentes et toujours actuelles sur les politiques génocidaires, sur les menaces du néonazisme et du révisionnisme. "Gardien de la mémoire d'Auschwitz», Primo Levi livre un message politique essentiel: "l'homme est et doit être sacré pour l'homme partout et à jamais».

Ce riche tour d'horizon sur les rapports entre politique et mort est illustré par des œuvres de Michèle Lepeer. Cette artiste partage ses réflexions sur son parcours dans le cadre d'un entretien avec sa fille. Les perspectives retenues dans ce numéro s'articulent autour de quatre pôles : pouvoirs et résistances; châtiments, violences et guerres ; rituels, valeurs et entretien de la mémoire ; enfermements, génocides et ethnocides. Ces thèmes représentés visuellement témoignent des multiples imbrications et interférences entre le politique et la mort, qu'illustrent à profusion les conflits et les dangers qui menacent notre planète, et dont certains ont ici fait l'objet d'analyses fouillées. 